References:

Barkov, Y.A. et al. (2002). Can Mineral 40, 671-678.

Sluzhenikin, S.F. \& Mokhov, A.V. (2015). Mineralium Deposita 50, 465-492.

Weihrich, R. et al. (2007). Prog Solid State Chem 35, 309-327.

Keywords: crystal structure, new minerals, sulphides
MS15-P03

\section{Carbon molecules in space: a thermal equation of state study of solid hexamethylentetramine}

Giulia Novelli1 ${ }^{1}$, Helen Maynard-Casely², Konstantin

Kamenev $^{1}$,William G. Marshall ${ }^{3}$, Garry McIntyre ${ }^{2}$, Simon Parsons ${ }^{1}$

1. School of Chemistry and Centre for Science at Extreme Conditions, University of Edinburgh, Edinburgh, United Kingdom

2. Australian Nuclear Science and Technology Organisation, Sydney, Australia

3. ISIS Neutron and Muon Facility, Oxford, United Kingdom

email: s1794786@ed.ac.uk

Equations of State (EoSs) show how the thermodynamic variables of temperature $(\mathrm{T})$, pressure $(\mathrm{P})$ and volume $(\mathrm{V})$ are inter-related. The ideal gas law, $P V=n R T$, is an example of an EoS which is used as a simple but effective model to explain the properties of gases. EoSs can also be applied to solids, where they can be used to show how energy changes when the solid experiences dramatic changes in its environment. Such information is relevant to understand the fate of carbon in the context of planetary settings, from comets to gas giants. Despite the large heterogeneity of galactic and interstellar regions, the organic chemistry of the universe seems to follow common pathways. Molecules of high astrobiological relevance such as N-heterocycles and amino acids have been identified in trace quantities in meteorites but they can be considered fragile species easily destroyed by raditions, shocks and thermal processes through the Solar System.

Alhough it is quite common to model thermal expansion at ambient pressure with a VT equation of state (EoS), and compression using a PV EoS, determinations of PVT EoSs are much less common, particularly for molecular materials. The paucity of data reflects the difficulty in varying pressure and temperature simultaneously in crystallographic experiments, especially at reduces temperatures. These difficulties are addressed by the variable temperature insert for the Paris-Edinburgh cell available on the PEARL instrument at the ISIS Neutron Spallation Source.

A PVT EoS provides access to a rich array of thermodynamic properties which revel the changes in different contributions to the thermodynamic properties such as internal energy, enthalpy and entropy occur in response to compression. The same quantities can be modelled using computational methods such as the PIXEL method and Density Function Theory, making EoS measurements sensitive tests of theory. This poster will describe a PVT EoS determination for hexamethylentetramine- $d_{12}$ a high-symmetry crytalline solid which remains in the same phase from 130 to $480 \mathrm{~K}$ and between ambient pressure and $5 \mathrm{GPa}$.

\section{References:}

[1] P. Ehrenfreund and M. A. Sephton. (2006). Faraday Discussions, $133,277-288$

[2] S. C. Capelli, A. Albinati, S. A. Mason and B. T. M. Willis. (2006). The Journal of Physical Chemistry A, 110, 11695-11703.

[3] S. Gärtner, B. Gundlach, T. Headen, J. Ratte, J. Oesert, S. Gorb, T. G. A. Youngs, D. T. Bowron, J. Blum and H. J. Fraser. (2017). The Astrophysical Journal, 848, 1-9. 